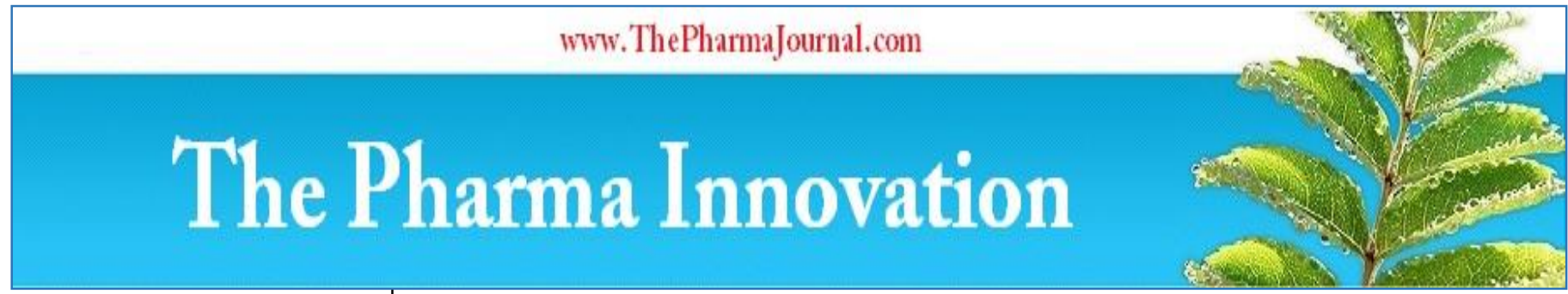

ISSN (E): 2277 - 7695

ISSN (P): 2349-8242

NAAS Rating: $\mathbf{5 . 2 3}$

TPI 2021; 10(6): 466-471

(C) $2021 \mathrm{TPI}$

www.thepharmajournal.com

Received: 17-04-2021

Accepted: 29-05-2021

\section{Mehjebin Rahman}

Department of Plant Pathology, Assam Agricultural University,

Jorhat, Assam, India

\section{Munmi Borah}

Department of Plant Pathology,

Assam Agricultural University,

Jorhat, Assam, India
Corresponding Author: Mehjebin Rahman

Department of Plant Pathology, Assam Agricultural University,

Jorhat, Assam, India

\section{Phytochemicals: Their role and mechanism in suppressing plant pathogenic bacteria}

\author{
Mehjebin Rahman and Munmi Borah
}

DOI: $\underline{\text { https://doi.org/10.22271/tpi.2021.v10.16g.6560 }}$

\section{Abstract}

Bacteria are unicellular prokaryotic micro-organisms which may be beneficial or harmful to plants. Earlier the infections caused by plant pathogenic bacteria were controlled by applying antibiotics and synthetic chemicals. But over-use of these antibiotics has resulted in evolving of antibiotic-resistant bacterial strains. Thus, it is the need of hour to explore alternative ways to target the bacterial virulence factors. Phytochemicals are extract from plants capable of inhibiting the growth of micro-organisms through various mechanisms, some which already been explained, while some others yet to be. In this review article, we are discussing about various phytochemicals, their source plant, their properties and composition, mode of actions, the bacterial phytopathogens on which they are effective, also some new perspectives of how they are gaining importance in the world of organic pesticides.

Keywords: Phytochemicals, plant disease management, bacterial infections

\section{Introduction}

Bacterial infections are one of the leading causes of infectious diseases in plants. There are 14 bacterial genera which include plant pathogenic bacteria: Erwinia, Pectobacterium, Pantoe, Agrobacterium, Pseudomonas, Ralstonia, Burkholderia, Acidovorax, Xanthomonas, Clavibacter, Streptomyces, Xylella, Spiroplasma, and phytoplasma. These opportunistic pathogens make their entry into host plants through stomata, lenticels, hydathodes or directly through wounds. The ability to form biofilms confers a selective advantage for bacteria to strive under harsh environmental conditions. Biofilms comprise multicellular assemblies of bacteria embedded in a complex extracellular matrix of exopolysaccharides and proteins. The formation of biofilms enables bacterial populations to adhere to environmental surfaces, including plant tissues, and is an intrinsic component of plant-microbe interactions and enables to perform different functions viz., quorum sensing, production of toxins for induction of disease, production of phytohormones, nitrogen acquisition, mobilization or enhanced uptake of soil minerals such as phosphorus, provide resistance to antimicrobial agents.

Plants produce a high diversity of natural products or secondary metabolites with a prominent function in the protection against predators and microbial pathogens based on their toxic nature and repellence to herbivores and microbes. Although Several plant secondary metabolites and their derivatives have been identified as possible antimicrobial agents and a promising field in resistance modifying agents to mitigate the spread of bacterial resistance. Among the secondary metabolites studied, alkaloids and polyphenols have shown strong antimicrobial activity. Isolated secondary metabolites are estimated to be $<10 \%$ of the total number available in plants.

Phytochemicals as antibacterial alternatives to promote growth and enhance host health Phytochemicals are also referred to as phytobiotics or phytogenics, as these chemicals are natural bioactive compounds derived from plants and are used to incorporate into plants and animals to enhance productivity. For being an ideal antibiotic alternative, the main aim where we need to focus is to have the same beneficial effects of AGPs which increase nutrient availability. Phytochemicals are used in solid, dried and ground form or as extracts (crude or concentrated). They are classified as essential oils (Eos); volatile lipophilic substances obtained by cold extraction or steam/alcohol distillation) and oleoresins (extracts derived by non-aqueous solvents) depending on the process used to derive the active ingredients. The main bioactive compounds of the phytochemicals are polyphenols, and their composition and concentration vary according to the plant, parts of the plant, geographical origin, harvesting season, environmental factors, storage conditions, and processing techniques. 
In recent years, phytochemicals have been used as antibacterial and antifungal agents for controlling plant diseases as well as natural growth promoters in plants.

\section{Some important phytochemicals successful in suppressing bacterial phytopathogens}

Plants produce several secondary metabolites which have antimicrobial properties against a wide range of pathogenic microorganisms. These compounds are constitutive and exist in healthy plants in their biologically active forms. They elicit chemotherapeutic or chemoprophylactic properties when encounter any infectious enteric diseases. Many mechanisms of antimicrobial action of phytochemicals have been suggested by different researchers but there still exists some uncertainties as to the molecular basis for their modes of action. Some phytochemicals may act by inhibiting microbial growth, inducing cellular membrane perturbations, interference with certain microbial metabolic processes, modulation of signal transduction or gene expression pathways. Although these are remarks from preliminary research, and the molecular basis for the modes of action of plant-based antibiotics needs to be ascertained.

\section{a) Polyphenols}

Phenolic compounds are plant secondary metabolites that constitute one of the most common and widespread groups of substances in plants. As stated by Harborne, the term "phenolic" or "polyphenol" can be precisely defined chemically as a substance which possesses an aromatic ring bearing one(phenol) or more (polyphenol) hydroxyl substituents, including functional derivatives (esters, methyl ethers, glycosides, etc.): as a general rule, the terms phenolics and polyphenols refer to all secondary natural metabolites arising biogenetically from the shikimate-phenylpropanoidsflavonoids pathways producing monomeric and polymeric phenols and polyphenols. Unless they are completely esterified, etherified or glycosylated, plan tphenolics are normally soluble in polar organic solvents. Most phenolic glycosides are water-soluble but the corresponding aglycones are usually less. With a few exceptions, water solubility increases with the number of hydroxyl groups present. Some phenolics are solubilized in sodium hydroxide and sodium carbonate but in alkaline medium their oxidation is enhanced and therefore treatment with alkaline solvents should either be performed under N2or preferably avoided. Phenolics with only few hydroxyl groups are soluble in ether, chloroform, ethyl acetate, methanol, and ethanol. Methanol, ethanol, water, and alcohol-water mixtures are most used for dissolving phenolic compounds for analytical purposes.

Plants need phenolic compounds for pigmentation, growth, reproduction, resistance to pathogens and for many other functions. Generally, the role of phenolic compounds in defence is related to their antibiotic, antinutritional or unpalatable properties. Phenolics-based plant defense mechanisms include physical changes such as lignifications and suberization of the plant cell walls (Stein et al., 1993), as well as metabolic changes such as de novo synthesis of PR proteins (Carr\& Klessig, 1989), and biosynthesis an daccumulation of phenylpropanoid secondary metabolites (Bennett \& Wallsgrove, 1994).

Salicylic acid (SA), a simple phenolic compound, is the signaling molecule required for induction of the systemic acquired resistance in plants (Durrant and Dong, 2004). Recently, it has been discovered that novel phenolic compounds $o$-coumaric acid (OCA), $t$-cinnamic acid (TCA), and $p$-coumaric acid (PCA) modulate the expression of T3SS genes in Dickeyadadantti, a broad-host range phytopathogen (Li et al., 2009; Yang et al., 2008). Some of their derivatives were found to suppress T3SS gene expression in Pseudomonas aeruginosa and Erwinia amylovora (Yamazaki et al., 2012; Khokhani et al., 2013). Pre-treatment of Xoowith TS006 (o-coumaric acid, OCA), TS010, TS015, and TS018 resulted in significantly attenuated HR without affecting bacterial growth or survival. These inhibitors reduced disease symptoms of both Xo oand Xoc of rice. Total phenolic compounds, free and total salicylic acid (SA), and enzymes related to plant defense, i.e., polyphenol oxidase (PPO) and catalase (CAT) are effective against Ralstonia solanacearum. Protocatechualdehyde (protocatechnic aldehyde, PCA) is a natural polyphenol compound that is isolated from the roots of traditional Chinese medicine radix Salviae Miltiorrhizae which is also effective in inhibiting $R$. solanacearum.

\section{b) Terpenoids}

Terpenoids are the largest group of plant specialized (secondary) metabolites and consist of five carbon isoprene units which are assembled to each other by thousands of ways. Terpenoids are modified terpenes, wherein methyl groups have been moved or removed, or oxygen atoms added. Their basic backbone structures are synthesized by the enzyme terpene synthase (TPS), following which they are further modified by, for example, hydroxylation, dehydrogenation, acylation or glycosylation (Dudareva et al. 2004, Pichersky et al. 2006) [17], resulting in an array of chemically diverse terpenoid compounds. Just like terpenes, the terpenoids can be classified according to the number of isoprene units that comprise the parent terpene:

- Hemiterpenoids, 1 isoprene unit (5 carbons)

- Monoterpenoids, 2 isoprene units (10C, i.e., derived from monoterpenes)

- Sesquiterpenoids, 3 isoprene units (15C, i.e., derived from sesquiterpenes)

- Diterpenoids, 4 isoprene units (20C, i.e., derived from diterpenes). ginkgolides.

- Sesterterpenoids, 5 isoprene units (25C, i.e., derived from sesterterpenes).

- Triterpenoids, 6 isoprene units (30C, i.e., derived from triterpenes). Example: sterols.

- Tetraterpenoids, 8 isoprene units (40C, i.e., derived from tetraterpenes). Example: carotenoids)

- Polyterpenoid with a larger number of isoprene units

Terpenoids can also be classified according to the number of cyclic structures they contain.

Terpenoids represent a large group of phytochemicals with promising antimicrobial activity Carbonylation of terpenoids was believed to increase bacteriostatic activity but not necessarily the bactericidal activity. A bacteriostatic agent is an agent that stops or inhibits microbial growth, while a bactericidal is responsible for killing the microbe. Monotepenes (carvacrol, thymol, menthol, and geraniol) possess antimicrobial activity against Gram-positive and Gram-negative bacteria by become Sng a potent efflux pump inhibitor. Phenol monoterpenes, such as carvacrol were also reported to inhibit biofilm development. Dihydro- $\beta$ agarofuran sesquiterpenes, namely $1 \alpha$-acetoxy- $6 \beta$, $9 \beta$ dibenzoyloxy-dihydro- $\beta$-agarofuran inhibits the growth of Bacillus spp. Essential oils containing Terpenoids from 
Clove, Oregano, Thyme are effect against plan pathogenic bacteria Burkholderiacepaci, from Lavender and Pine against Pseudomonas spp. from Cedarwood against Bacillus spp. Xanthomonas campestris pv. phaseoli (causing blight of bean), Clavibactermichiganensis subsp. michiganensis (bacterial wilt and canker of tomato), and Pseudomonas tolaasii (causal agent of bacterial brown blotch on cultivated mushrooms) are inhibited by Carvacrol which is the major constituent of Winter green and geranial and neral which are the major constitiuents of lemongrass. C. aurantium, C. aurantifolia Eos, and their major constituents including citral, linalool, citronellal, geraniol, _-terpineol, and linalyl acetate indicated antibacterial effects against Xcc. Monoterpenes $\alpha$ thujene, $\alpha$-pinene, sabinene, myrcene, $\alpha$-terpene, and (S)limonene and sesquiterpenes cyclosativene, $\alpha$-copaene, and $\beta$ elemene were detected from 1-week-old Xoo-infected rice seedlings. All monoterpenes were constitutively released from rice seedlings before Xoo infection. But (S)-limonene emission was further elicited after exposure of the seedlings to Xoo in coincidence with upregulation of limonene synthase gene (OsTPS20) transcripts. Only the stereospecific (S)limonene [and not (R)-limonene or other monoterpenes] severely inhibited Xoo growth, as confirmed by disc diffusion and liquid culture assays. Rice seedlings showed suppressed pathogenic symptoms suggestive of resistance to Xoo infection after foliar treatment with (S)-limonene. Thus, (S)limonene is a volatile phytoanticipin, which plays a significant role in suppressing Xoo growth in rice seedlings.

\section{c) Alkaloids}

Alkaloids factually or literally means alkali-like, possessing a nitrogen atom with an unshared pair of electrons. They are mostly solids except those that lack oxygen are liquids. They are insoluble or sparingly soluble in water, unless reacted with an acid to form a salt. Alkaloids are soluble in non-polar solvents like chloroform, but their salts are not. They are a large and structurally diverse group of natural products of microbial, plant, and animal origin which consist of antimicrobial properties. They occur in around 300 plant families though specific compounds confined to certain families only. Eg: hyoscyamine is found only in Solanaceae family of plants. Alkaloids can occur in any part of the plant, though specific compounds may be limited to a certain part (eg. quinine in cinchona tree bark). The active compounds budmunchiamine-A and pithecolobine extracted from $A$. amara and $A$. saman have broad spectrum bactericidal activity against Xanthomonas. Alkaloid extracts of $E$. citriodora and $R$. communis have antimicrobial activity and are potential as active ingredient for preparation of biocide formulations in controlling bacterial wilt disease of plants.

d) Phytosterols

Phytosterols are an important family of lipids present in plant cells which encompass sterols, stanols and steroids. They vary only in carbon side chains and presence or absence of double bond. Stanols are saturated sterols, having no double bonds in the sterol ring structure. Free phytosterols extracted from oils are insoluble in water, relatively insoluble in oil, and soluble in alcohols. Phytosterols can be classified either as 4desmethylsterols, 4-methylsterols, or 4,4'-dimethylsterols. Sitosterol, campesterol, and stigmasterol (4-desmethylsterols group) are found in abundance in most of the plants. However, there are over 200 different sterol structures that have been discovered in various plant species. Sterols may be found in free form (free sterols, FSs) or as conjugated steryl esters (SEs), steryl glycosides (SGs) and acyl steryl glycosides (ASGs). Conjugated sterols are ubiquitously found in plants, but their relative contents highly differ among species and their profile may change in response to developmental and environmental cues. Sitosterol regulates membrane fluidity, permeability, modulate the activity of membrane-bound enzymes and helps plant adapt to temperature and plant immunity against pathogens.

In Solanaceous plants, e.g., potato (Solanum tuberosum), cholesterol production rises to match the demand for the synthesis of steroid glycoalkaloids in response to wounding or pathogen infection (Arnqvist et al., 2003). Similarly, pathogenic bacteria and reactive oxygen species stimulate the biosynthesis of stigmasterol in Arabidopsis. Indeed, the overexpression of one of the Arabidopsis stigmasterol biosynthesis genes resulted in enhanced resistance to bacterial pathogens (Wang et al., 2012). Again, Pathogenesis-Related Protein 1 (PR-1) can bind sterols including stigmasterol in vitro. PR-1 inhibits pathogen growth by sequestering sterols from pathogens.

Bacterial pathogens colonize a host plant by growing between the cells by utilizing the nutrients present in apoplastic space. When pathogenic bacteria attack their host plants, bacterial PAMPs trigger a general plant defense response, leading to induction of stigmasterol through PTI and/or ETI. This restricts nutrient transfer from cytosol to the apoplast thus inhibiting colonization of the pathogenic bacteria. Expression of the AtCYP710Al gene in Arabidopsis plant is responsible for sterol biosynthesis which increases during both compatible and incompatible interaction with pathogens. AtCYP710Alis a plant defense gene and its over-expression might confer disease resistance to host pathogens.b-sitosterol and stigmasterol detected in grape pomace extracts are used as an antibacterial agent against Xanthomonas citri.

\section{e) Organosulphur compounds}

Sulphur containing secondary compounds of plant origin are known to have antimicrobial utility. Natural products that have sulphur-compounds constitute a formidable wall of defense against a wide range of pathogens and pests. The history of crop protection is closely linked to elemental sulfur, which is undoubtedly the oldest of all pesticides. Its fungicidal properties were already known by the ancient Greeks, when nearly thirty centuries ago Homer referred to "pest-averting sulfur". Most of the knowledge was lost until Forsyth re-discovered sulfur for the control of plant diseases in 1802. His recommendation for the control of powdery mildew on fruit trees was a concoction of quick-lime, sulfur, elderberry bud and tobacco. Since 1824 powdered sulfur was widely applied against fruit and grape diseases, 4 such as peach powdery mildew, until the second half of the 19th century saw the introduction of three further pioneering sulfur-containing fungicides: Eau Grison (calcium polysulfide) in 1852, Bordeaux mixture $\left[\mathrm{CuSO}_{4}+\mathrm{Ca}(\mathrm{OH})_{2}\right]$ in 18856 and Burgundy mixture $\left(\mathrm{CuSO}_{4}+\mathrm{Na}_{2} \mathrm{CO}_{3}\right)$ in 1887. These inorganic sulfur derivatives are still in operation, but today's typical agrochemicals are organic compounds. Because of the significance of sulfur in modern organic chemistry and the diverse biological activities of the resulting compounds, approximately $30 \%$ of all registered crop protection agents contain at least one sulfur atom.

The role of sulfur (S) in the resistance of crops against diseases became obvious at the end of the 1980s. Among the sulfur-containing secondary metabolites, two groups of 
compounds are involved in activated plant defense systems, the glucosinolates and the alliins. In the intact plant tissue, these compounds, which are relatively physiologically inert by themselves, are spatially separated from their hydrolyzing enzymes, the myrosinases and alliinases, respectively. When the tissue is damaged, for example upon microbial attack, the parent compounds are converted to biologically active products by the action of the hydrolyzing enzymes. The precursors as well as the activating enzymes are usually stored in very high amounts so that a fast release of sufficient amounts of hydrolysis products is ensured.Sulfur-containing metabolites that are supposed to be involved in pathogen resistance are glutathione, glucosinolates, the gaseous release of volatile S, phytoalexins, S-rich proteins, and the formation of elemental S.Allicin (diallylthiosulfinate) is the volatile, organosulfur, prooxidant compound extracted from garlic (Allium sativum L., Amaryllidaceae) tissues. The thiolsulfinate-compounds, like their allyl-relatives, show diverse biological activities, like strong antibacterial properties. Eg: Petivericin from Petiveriaalliacea. Brassicaceae, along with 16 other plant families in the order Brassicalescontain glucosinolates, the greatest diversity being found in the Capparaceae, Resedaceae and Tovariaceae. Thiophenes in Asteraceae are heterocyclic aromatic sulfurcompounds called thiophenes $(\mathrm{C} 4 \mathrm{H} 4 \mathrm{~S})$ are important phytoanticipinsthat are commonly known to be associated with defence against bacterial pathogens. Elementary S plays an established key role in plant defence as exemplified by its detection in representative members of five different plant families namely tomato and tobacco (Solanaceae), cotton (Malvaceae), French bean (Leguminosae), wild cabbage (Brassicaceae), and cocoa (Sterculiaceae). Elemental sulfur's role in induced plant defence was amply demonstrated by its formation (a) in xylem tissue of disease resistant, but not susceptible tomato against R. solanacearum. However, sulfur was not found either in the xylem of $V$. dahliae-infected strawberry or in the leaves of six plantspecies including tomato, cabbage, tobacco and French bean hypersensitive to pathovars of Pseudomonas syringae, thussuggesting that it is not produced in all plant species and that its induced response in plants may be xylem-specific.SThe sulfurcontainingindolethiazole compound camalexin (3-thiazol-2'-ylindole)is the major phytoalexin of the model plant Arabidopsisthaliana (L.) Crantz.. Camalexin was originally found inCamelina sativa (Brassicaceae). Tsjuiet al. reported in 1991that infection with Pseudomonas syringaepv. syringaeresulted inthe accumulation of a phytoalexin and named it "arabilexin". For bacteria it is known that camalexin's toxicitiy is due to disruption of cell membrane.

\section{Antibacterial Activity of Phytochemicals from Medicinal Plants}

Medicinal plants viz., Althea officinalis L. (Malvaceae), Origanum vulgare Oregano (Lamiaceae), Plantago lanceolata L. (Plantaginaceae), Polygonum bistorta L. (Polygonaceae), Satureja hortensis L. (Lamiaceae), Solanum dulcamara L. (Solanaceae), and Quercus robur L. (Fagaceae), have antibacterial activity by against important phytopathogenic bacteria Xanthomonas pathovars viz., Xanthomonas axonopodis pv. malvacearum, Xanthomonas axonopodis pv. phaseoli and Xanthomonas campestris pv. vesicatoria associated with angular leaf spot of cotton, common blight of beans and bacterial spot of tomato respectively. The inhibitory effect of these medicinal plants is due to the presence of phenolic and acidic fractions. The Indian medicinal plants: Achyranthes aspera, Artemisia parviflora, Azadirachta indica, Calotropis gigantean, Lawsonia inermis, Mimosa pudica, Ixora coccinea, Parthenium hysterophorus and Chromolaena odorata are found to be effective against Xanthomonas vesicatoria and Ralstonia solanacearum. Again, essential oils from oregano, sweet-flag, caraway, peppermint, common, fern leaf and willow-leaved yarrow field were investigated against growth of phytopathogenic bacteria. Xanthomonas vesicatoria Pseudomonas spp. strains and Erwinia carotovora.

Table 1: Few examples of major phytochemical compounds extracted from plants and showed potent antibacterial activity.

\begin{tabular}{|c|c|c|c|}
\hline Plant Name & Scientific name & Phytochemical category & ssAgainst plant bacteria \\
\hline Oil cake tree & Albizia amara, & Budmunchiamine-A (Alkaloids) & Xanthomonas spp \\
\hline Rain tree & Albizia saman & Pithecolobine (Alkaloids) & Xanthomonas spp \\
\hline Tomato & Solanum lycopersicum & DL-3-aminobutyric acid (BABA) (Polyphenolsa) & Ralstonia solanacearum \\
\hline Rice & & Salicylic acid (Polyphenols) & Xanthomonas oryzae oryzae \\
\hline Mustard & Brassica rapa & $\begin{array}{l}\text { Gluconapin and its isothiocyanate (Glucosinolates } \\
\text { and phenolic compounds) }\end{array}$ & Xanthomonas campestris \\
\hline Citrus & $\begin{array}{l}\text { Citrus aurantium, } C . \\
\text { aurantifolia, } \\
\text { Fortunella } \mathrm{sp}\end{array}$ & $\begin{array}{c}\text { Citral, Linalool, Citronellal, Geraniol, _-Terpineol, } \\
\text { and Linalyl acetate }\end{array}$ & Xanthomonas citri \\
\hline $\begin{array}{l}\text { Wintergreen, } \\
\text { oregano, } \\
\text { lemongrass }\end{array}$ & $\begin{array}{l}\text { Gaultheria procumbens, } \\
\text { Origanum vulgare, } \\
\text { Cymbopogon flexuosus }\end{array}$ & Carvacrol, Geranial, Neral (Terpenes) & $\begin{array}{c}\text { Xanthomonas campestris pv. } \\
\text { phaseoli, Clavibacter michiganensis } \\
\text { subsp. michiganensis }\end{array}$ \\
\hline
\end{tabular}

New Perspectives on the Use of Phytochemicals as an Emergent Strategy to Control Bacterial Infections

Phytopathogens are Gram-negative bacteria such as Pseudomonas, Acinetobacter, Escherichia, and Enterobacter spp. They are difficult to control due to their nosocomial status and expression of MDR phenotypes, which makes the treatment of majority of current infectious diseases with antibiotics less effective. The effectiveness of traditional antibiotics is decreasing in bioactivity due to the emergence of MDR bacteria, which makes it imperative to search for alternative treatments. Only two classes of synthetic antibiotics were developed in the past 50 years: fluoroquinolones and oxazolidinones (linezolid). All other similar efforts to find a new antibiotic failed, which indicates that there is an extremely low probability of discovering a new drug.

These have triggered the importance of looking for some other alternatives to control the bacterial infections in plants. Plants can recognize pathogen signals or elicitors and activate immune responses through the reinforcement of the cell wall, the biosynthesis of lytic enzymes, and the production of secondary metabolites and pathogenesis-related proteins. 
These detective compounds in plants are either constitutive, stored as inactive forms, or inducible in response to pathogen attack. The former is known as phytoanticipins and the latter as phytoalexins. Phytoanticipins include saponins, cyanogenic glycosides and glucosinolates. They are present in the plant before microorganism attack or produced after infection from pre-existing precursors. Some phytoanticipins are found at the plant surface while others are present in vacuoles or organelles and are released through a hydrolyzing enzyme after pathogen challenge. Phytoalexins include terpenoids, glycosteroids, flavonoids and polyphenols. These are small molecules (molecular weight $<500$ ) which are both synthesized and accumulated in the plant after the recognition of elicitors derived from exposure to attackers.

The degree of severity and the persistence of bacterial infections are worsened when microorganisms form biofilms. There are different stages of biofilms Development e.g., adhesion, motility, production of extracellular polymeric substances (EPS) and QS phenomena and inducing biofilm inactivation. Biofilms removal can be promising by strategies like weakening, dispersion or disruption. These strategies were fulfilled by adopting several means: using surfactants/biosurfactants and other types of non-antibiotic coatings, blocking of the adhesins' interaction with their receptors, use of chelating agents that inhibit the transport of essential metals to the interior of cells, thus stopping biochemical pathways that are crucial for biofilm formation, Interference with the bacterial communication through the use of QS inhibitors (QSI); the use of non-pathogenic bacteria which can compete with pathogens by producing toxins (e.g., bacteriocins) or other substances, thus preventing colonization, Induction of dispersal by the application of enzymes, The use of bacteriophage, Eradication of persistent cells (e.g., combined application of sugars or silver with antibiotics and/or an increase in the production of reactive oxygen species), application of ultrasounds, electrical fields and photosensitizers, nanoparticles, polymer-based antimicrobials, liposomes and aerosols. Finally, natural compounds from plants have drawn the attention of as they are considered a green and sustainable source of new molecules that have shown to be effective biofilm inhibitors. The structural diversity displayed by phytochemicals associated with their multi-target mode of action is differs significantly from the properties of conventional antibiotics. These distinctive properties can help to overcome the resistance problem in case of antibiotics. In fact, there is no evidence on the occurrence of bacterial resistance to phytochemicals.

\section{Phytochemicals as biofilms metal chelators}

In addition to the key roles that metals play in plants and microorganism, metals play their role in pathogenesis and virulence. They are associated with biofilms formation, s they serve as signaling factors. Iron sensing is mainly involved in the first steps of biofilm formation as they are vital for cell growth and adherence. Iron also helps to release DNA, one of the major biofilm's matrix components in P. aeruginosa. Iron and calcium are emphasized for their crosslink properties among components of the biofilm's matrix, helping the maintenance of the matrix integrity. Plant-derived compounds with chelating properties are polyphenols, phenolic compounds, and flavonoids with 6, 7-dihydroxy iron chelating site.

\section{Conclusion}

There is an increasing concern about the severity of bacterial infections in crops while there are only a limited number of antibiotics in the market. The agricultural fields need wide range of antibiotics. Since there are only a limited number of antibiotics, farmers have been compelled to use the same antibiotics repeatedly which resulted in development of resistance in bacteria. A novel and promising approach to deal with multidrug resistance is to improve the various antibiotics by employing active molecules capable of restoring antibiotic susceptibility in the pathogens. The design of such combination is a promising alternative that considers the scarcity of new and effective therapeutic antibacterial, especially against Gram-negative bacteria. Plants have been explored comprehensively as potential sources of EPIs and other antibiotic adjuvants. There have been numerous publications describing the isolation and purification of plantderived antimicrobials. The known inhibitors, however, are mostly active against MDR pumps of Gram-positive species; efforts to find inhibitors of Gram-negative species have not been successful so far. Advances in the understanding of the biofilm formation and resistance mechanisms are significant. However, the progress on the development of strategies for their effective control is modest. Among the promising strategies, the use of phytochemicals has attracted significant attention, as they can be used as a source of new scaffolds for the development of novel therapeutic compounds with enhanced activity; they can also target bacteria using different mechanisms of current antibiotics. Another interesting aspect of the use of phytochemicals against biofilms is their ability to influence regulatory mechanisms involved in biofilm formation and maintenance by a non-bactericidal mode of action, and thus they do not impose selective pressure upon bacteria. However, caution in the application of these antivirulence approaches, such as the inhibition of QS pathways, is needed. In fact, an important missing piece from the studies of plant-derived antimicrobial/antibiofilm compounds is the understanding of their mode of action. Currently, this method that makes the treatment of biofilms infections possible is the administration of several antimicrobial agents at high doses and during prolonged periods. Indeed, so far, there are no identified compounds with total efficacy against biofilms. To surpass this drawback the combined application of several strategies will allow targeting different biofilms sites.

\section{References}

1. Bhardwaj, Surender, Singh Jitender. Antibacterial activity of some plant extracts against plant pathogenic bacteria Xanthomonas campestris pv. campestris. Indian J Agric Res. 2009, 43.

2. Cushnie, Tim, Cushnie, Benjamart, Lamb, Andrew. Alkaloids: An overview of their antibacterial, antibioticenhancing and antivirulence activities. International Journal of Antimicrobial Agents 2014.

3. Mahizan Nik, Yang, Shun Kai, Moo, Chew-Li, Song Adelene et al. Terpene Derivatives as a Potential Agent against Antimicrobial Resistance (AMR) Pathogens. Molecules 2019.

4. Thawait, Lokesh, Mahatma, Mahesh, Kalariya, Kuldeepsingh et al. Plant Phenolics: Important BioWeapon against Pathogens and Insect Herbivores. Popular Kheti. 2014;2:149-152.

5. Hernández-Romero, Diana, Solano, Francisco, Sanchez- 
Amat, Antonio. Polyphenol Oxidase Activity Expression in Ralstonia solanacearum. Applied and environmental microbiology 2005.

6. Velasco, Pablo, Lema M, Francisco, Marta, Soengas Pilar et al. In vivo and in vitro Effects of Secondary Metabolites against Xanthomonas campestris pv. campestris. Molecules (Basel, Switzerland) 2013.

7. Fan, Susu, Tian, Fang, Li, Jianyu et al. Identification of phenolic compounds that suppress the virulence of Xanthomonas oryzae on rice via the type III secretion system: Anti-virulence compounds for Xanthomonas oryzae. Molecular Plant Pathology 2016.

8. Hassan, Moahmed, Abo-elyousr, Kamal. Activation of tomato plant defence responses against bacterial wilt caused by Ralstonia solanacearum using DL-3aminobutyric acid (BABA). European Journal of Plant Pathology 2012.

9. Todorović, Biljana, Potocnik, Ivana, Rekanovic, Emil et al. Toxicity of twenty-two plant essential oils against pathogenic bacteria of vegetables and mushrooms. Journal of Environmental Science and Health, Part B. 2016;51:1-8.

10. Golmohammadi, Morteza. The Effect of Citrus Essential Oils and Their Constituents on Growth of Xanthomonas citri subsp. citri. Molecules Online 2017, 22.

11. Koche, Deepak. Role of secondary metabolites in plants' defense mechanism 2018.

12. Yazaki, Kazufumi, Arimura, Gen-Ichiro, Ohnishi Toshiyuki. "Hidden" Terpenoids in Plants: Their Biosynthesis, Localisation and Ecological Roles. Plant \& cell physiology 2017.

13. Lillehoj, Hyun, Liu, Yanhong, Calsamiglia, Sergio et al. Phytochemicals as antibiotic alternatives to promote growth and enhance host health. Veterinary Research. 2018

14. Borges A, Abreu AC, Dias C, Saavedra MJ, Borges F, Simões M. New Perspectives on the Use of Phytochemicals as an Emergent Strategy to Control Bacterial Infections Including Biofilms. Molecules (Basel, Switzerland) 2016;21(7):877.

15. Babu, Shafeer, Satish S, Mohana, Devihalli, Mp Raghavendra, Anandarao et al. Antibacterial evaluation and phytochemical analysis of some Iranian medicinal plants against plant pathogenic Xanthomonas pathovars. J Agricultural Technol 2007, 3.

16. Sukanya S, Jogaiah, Sudisha, Hariprasad P, Niranjana S, Prakash HS et al. Antimicrobial activity of leaf extracts of Indian medicinal plants against clinical and phytopathogenic bacteria. African journal of biotechnology 2009.

17. Vasinauskienè M, Radusiene, Jolita, Zitikaitė I, Surviliené, Elena. Antibacterial activities of essential oils from aromatic and medicinal plants against growth of phytopathogenic bacteria. Agronomy Research 2006, 4

18. Camele, Ippolito, Elshafie, Hazem, Caputo, Lucia et al. Anti-quorum Sensing and Antimicrobial Effect of Mediterranean Plant Essential Oils Against Phytopathogenic Bacteria. Frontiers in Microbiology. 2019.

19. Sampietro, Diego, Lizarraga, Emilio, Ibatayev, Zharkyn, et al. Chemical composition and antimicrobial activity of essential oils from Acantholippia deserticola, Artemisia proceriformis, Achillea micrantha and Libanotis buchtormensis against phytopathogenic bacteria and fungi. Natural product research 2015.

20. Shaheen, Hanan, Issa, Marwa. In vitro and in vivo activity of Peganum harmala L. alkaloids against phytopathogenic bacteria. Scientia Horticulturae 2019, 264. 